\title{
Air Traffic Control Tools Assessment
}

\section{Tomáš Noskievič ${ }^{1}$, Jakub Kraus ${ }^{1 *}$}

\author{
${ }^{1}$ Department of Air Transport, Faculty of Transportation Sciences, Czech Technical University in Prague, Prague, \\ Czech Republic \\ *Corresponding author: Czech Technical University in Prague, Faculty of Transportation Sciences, Department of Air \\ Transport, Horská 3, 12803 Prague, Czech Republic, Email: kraus@ fd.cvut.cz
}

\begin{abstract}
Undoubtedly air transport in today's world wouldn't be able to exist without any air traffic control service. As the air transport has been coming through major changes and it has been expanding, it is assumed that its volume will be doubled in the next 15 years. Air traffic control uses strictly organised procedures to ensure safe course of air operations. With the skies covered with more airplanes every year, new tools must be introduced to allow the controllers to manage this rising amount of flying aircraft and to keep the air transport safe. This paper provides a comprehensive and organized material, which describes the newest tools and systems used by air traffic control officers. It proposes improvements for further research and development of ATC tools.

\section{Keywords}

Air Traffic Control - Arrival Manager - Departure Manager - Medium Term Conflict Detection — Satefy Nets - Trajectory Prediction
\end{abstract}

\section{Introduction}

To well understand the necessity of new systems, it is important to show how air traffic controllers work. Air traffic control is divided into Area Control (ACC), Approach Control (APP) and Tower Control (TWR). Controllers who work in ACC and APP work together in pairs. Each pair of controllers is responsible for one sector and consists of the planning (strategic) controller and the executive (tactical) controller.

The task of the planning controller is to communicate with other sectors and to coordinate all flights that are to enter his sector. The most important part of his task is to search for potential conflicts to decrease his colleague's workload. The executive controller communicates directly with pilots from the point they enter his sector until he hands them over to the next sector. He follows the aircraft on a radar screen and when it is necessary he issues clearances. The executive controller plays a key role in determining the sector capacity, as it is defined as a maximum number of aircraft that are allowed to enter an airspace during a given period of time together with allowing acceptable workload of the executive controller [1]. This is why the new tools to help controlling of air traffic flows are needed. With more and more flying aircraft we have to find new solutions to decrease the controller's workload. This paper introduces some major characteristics of:

- Medium Term Conflict Detection,

- Arrival Manager,

- Departure Manager,

- Safety Nets.

\section{The Most Important ATC Tools}

The current ATC environment uses 3D aircraft trajectories to follow planes. New tools should be much more accurate. To reach better accuracy the tools use $4 \mathrm{D}$ trajectories - time is the 
extra component. Knowing the fourth dimension enables predicting the future position of aircraft, better conflict searching and optimal controlling of approaching traffic nearby airports.

\subsection{Trajectory Prediction}

Developing new reliable algorithms for trajectory prediction is a tough nut. To get the most accurate calculation of the trajectories it is important to obtain information from many inputs. The system needs to know the aircraft performance data (these are obtained from EUROCONTROL's database called BADA), the weather forecast (predicted wind, temperature and pressure), the flight plan and routes. Finally, the system must know the definitions of the airspace in which it serves and the definitions of separation thresholds. The controllers can also input their clearances to the system and then the trajectory predictor calculates the best trajectory which is called the tactical trajectory as it is calculated not only based on the flight plan data, but also based on the last issued clearance.

\subsection{Medium Term Conflict Detection}

Medium Term Conflict Detection (MTCD) is a planning tool developed to monitor all aircraft in a given airspace. It examines trajectories in pairs and checks if they come close within a predefined separation limits. If so, an alert is displayed to controllers. The tool searches for aircraft-airspace conflicts as well as for aircraft-aircraft ones.

\subsubsection{MTCD development and trials}

The medium term conflict detection tool has been under development since half of 1990s. The first trials were held in 1998, nevertheless the results were highly unsatisfactory. Another simulations followed as a part of EATCHIP (The European ATC Harmonization and Integration Programme) in 2000. Airspaces of Paris ACC, Reims ACC and Maastricht UAC were used in the experiment. The aim of those simulations was to assess the impact of MTCD tool on the air traffic controllers and their confidence in the system. It was also important to find out whether the controller detected the same conflicts as the tool and vice versa - whether the tool detected the same conflict as the controller. The question if the tool can make the planning better and if it decreases the controller's workload should have been answered.

The participants evaluated the simulation as good and they adopted the new system quickly. Some of the detected conflicts were said not to probably happen in the real life as a better coordination with neighbouring sectors which were not included in the experiment would precede. The biggest problem which showed up were the false alerts which decreased the trust in the system. The simulation confirmed that MTCD provides the executive controller more time to solve the problems. The most problematic part of the whole system is the trajectory prediction.

The German provider of air traffic services Deutsche Flugsicherung $\mathrm{GmbH}$ was the first to implement the MTCD tool in 2011. It is a part of system VAFORIT in Karlsruhe control centre. This new system increased the capacity of the upper airspace by $11 \%$. Its development took a long time since 1996. The MTCD detects breaches of horizontal separation limits for a time horizon of 15 minutes - for now it provides hints where a conflict can possibly happen. Very high number of false alerts appeared after the system implementation, but the trajectory prediction algorithms were made better and currently there are less than $5 \%$ of false alerts and the controllers claim their satisfaction with the system is $90 \%$.

The British provider of air traffic services NATS also implemented a kind of MTCD system in 2011 in Swanwick ACC - iFACTS (interim Future Area Control Tools Support). This tool continuously monitor the development of all flights based on the flight plan data and the last issued clearance. The controller is immediately alerted when a plane deviates from the clearance. It is able to show the aircraft's trajectory up to 18 minutes based on the flight plan data. When the controller wants to change the flight level in which the plane flies, he can see a graphical representation of potential conflicts and check the decision before he issues the clearance.

\subsubsection{Description of Conflict Types}

The time horizon in which MTCD compares trajectories is according to EUROCONTROL Specification up to $20 \mathrm{~min}$ utes - it notifies the controller of interactions that occur in the medium-term [2]. Conflict is defined as a state when horizontal and vertical separations are predicted to be lost simultaneously [3].

The tool supports the controller in making decisions as it belongs to the category Decision Support Tools (DST) and it should assist the ATCO by providing [4]:

- information about all potential conflicts in predefined time horizon,

- time to assess the conflict,

- filtering which can be used to prioritise different problem types,

- information about the traffic which is conflicting,

- information about traffic that is not following ATCO's clearance,

- "what-if" probing to check whether a newly issued clearance will not result in conflict.

It is important to mention that despite all these MTCD functions the ATCO is still the one who is responsible for correct aircraft separation.

To predict the aircraft-aircraft conflict situations the system needs to obtain data from the trajectory prediction tool. Then the calculated trajectories are compared and assessed. When the separation threshold limits are breached, the controller is notified. For aircraft-airspace encounters, a database of defined airspace volumes must be included among the system inputs. Since MTCD is a geometric conflict detection tool, these encounters are revealed simply when the predicted trajectory infringes an airspace which the aircraft is not supposed to enter. The severity of the conflict is evaluated on 
the basis of the geometry of aircraft involved in the conflicting situation and the time available for ATCO to solve the problem.

Generally, MTCD searches for potential conflicts among all flights for which it has available data about their trajectories. New calculations are always made in these cases [5]:

- new trajectory is known to the system (new flight),

- a trajectory is updated,

- environment conditions change (weather changer, new restricted area),

- when a predefined time since last calculation has elapsed.

A conflict is detected when the distance between aircraft is less than a desired separation limit. Firstly, the tool examines the vertical separation. If it is right, no other calculations are made. If the vertical limit is breached, the horizontal segments which represent the probable positions of an aircraft for any given time, are examined. If the distance between these segments is less than required, another detailed calculation must be made to calculate the time remaining to the situation when the distance between the aircraft is the shortest and to calculate the shortest distance.

Conflicting situations between two aircraft are divided according to their cause. It could be either a tactical based conflict or a plan based conflict (see Fig. 1). The first case happens when the position of at least one of the aircraft involved is predicted from the tactical trajectory. The cause can be e. g. wrong ATC clearance or aircraft deviating from the last issued clearance. The second case is distinguished according to a position of the conflict origin: an entry problem and an exit problem. The entry problem is about to happen when the aircraft enters a new airspace. The exit problem is about to happen shortly before the aircraft should leave the current airspace. The planning controller is responsible for solving these conflicts. For instance, an unsolved entry problem can result in the fact that the executive controller does not have enough time to solve it. In-Sector problem also belongs to plan based conflicts. Its solution can be prepared and suggested to the executive controller by the planner.

A specific situation is when an ability to manoeuvre of one aircraft is limited by presence of other aircraft. For example, when one aircraft flies $2000 \mathrm{ft}$ above another one, the lower aircraft is unable to climb to higher flight level. This is a context problem.

The MTCD tool also checks if an aircraft infringes some restricted area. Holding patterns are a special case. Aircraft in higher flight levels use larger holding patterns because of higher speed. As they descent and the speed decreases, the pattern gets smaller. This is why holding areas should be defined in the MTCD system to check if traffic does not cross active holding pattern.

\subsection{Management of Arriving and Departing Traffic Flows}

\subsubsection{Arrival Manager}

The aim of the Arrival Manager (AMAN) is to provide the approach controller an assistance when controlling the flow of aircraft from defined entry point to approach fix or to runway threshold. It helps the controller to optimize the runway capacity and to manage the traffic flow entering TMA. It minimises the impact of air transport on the environment thanks to decreasing number of aircraft in holding patterns and less aircraft being vectored in low altitudes. The planning a sequencing function should reduce the controller workload, mainly in case of extraordinary situations, e. g. in case of closed runway.

This tool calculates the aircraft sequence from many inputs. Flight plan data are obtained from the Flight Data Processing System. The aircraft performance database is also needed. The system should also get the information about restricted airspaces and it must recognise different wake turbulence categories to make a safe sequence. Weather data, mainly concerning the wind, can be used for more accurate flight prediction.

Firstly, the trajectory prediction is very important to know when the aircraft enters the area of interest. The AMAN tool then calculates a first-to-come, first-to-serve sequence or a sequence based on defined requirements of the air traffic services provider. As output, the system shows a time-line of the counted sequence with information on recommended speed and time (whether the flight is delayed or it is to be landing early).

The tool is managed by the supervisor who sets landing rates, runways in use, closed runways. The approach sector controller is nevertheless responsible for tactical controlling of the aircraft, AMAN only supports the controller in planning.

\subsubsection{Departure Manager}

Managing the departing planes is important as well. Departure Manager (DMAN) works with estimated take off times and it takes into account the slot restrictions, runway in use and its capacity. The aim of this tool is to allow optimal runway capacity use, to plan and to organise the departing flows in TMA, to minimise delays and to allow a better coordination with area and approach controllers. With a combination with trajectory prediction and standard instrument departure routes database it can be used as a conflict detection tool in TMA. When there is only one runway available at the airport, it is necessary that DMAN cooperates with AMAN. AMAN provides the landing sequence and DMAN plans departures among the landing planes. DMAN calculates times when start up should be performed so that the aircraft does not spend much time waiting at the runway threshold.

The company Thales developed integrated AMAN and DMAN tool in a system called Maestro. It is in operation in Copenhagen, Nice and in Paris. A runway capacity grow by $10 \%$ was reached thanks to Maestro in Copenhagen. The system in Nice allowed to increase the landing rate from 26 to 


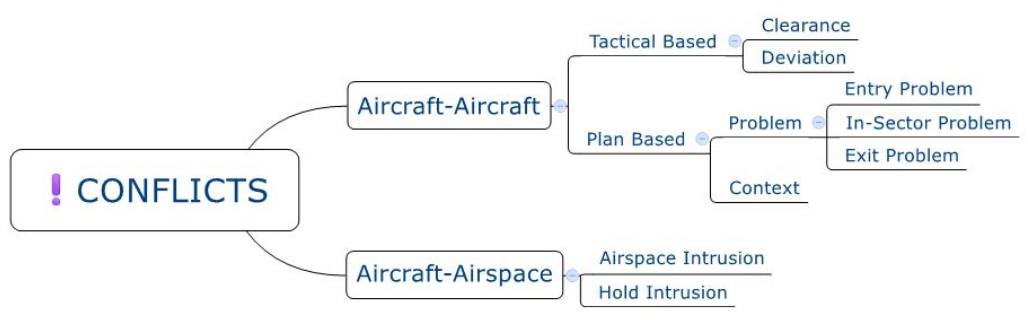

Figure 1. Scheme of conflicts types.

28 planes per hour. And finally, there was a rise in punctuality by $10 \%$ for departures in Paris CDG airport. The Maestro system is also used in Johannesburg, Sydney and Bangkok [6].

Although there are strict requirements on the whole air traffic control system, sometime even this system fails. This is the reason why to implement the safety nets which are supposed to alert controllers to an increased risk to flight safety [7]. Safety nets could be either ground-based or airborne, nevertheless this paper deals with the ground-based ones.

\subsubsection{Short Term Conflict Alert}

Short Term Conflict Alert safety net notifies the controller on a conflict which is about to happen in two minutes time horizon. The conflict is detected on the base of a linear trajectory prediction - the linear extrapolation of the aircraft 3D vector. By comparing these extrapolated trajectories it is found out whether the pair of aircraft is in conflict or not.

According to the STCA Specification, it is required to get less than $30 \%$ of false alerts. According to the air traffic controllers, this is too much for false alerts. Regarding the controller's experience, the optimal amount of false alerts is when there are less than $5 \%$ of them. But this requirement differs from one control centre to another and it depends on the installation settings of individual air traffic services providers. The most important thing of the STCA is that its alert always comes before the ACAS alert on the flight deck (see Fig. 2).

\subsubsection{Minimum Safe Altitude Warning}

The importance of minimum safe altitude warning (MSAW) is in its contribution to avoidance of controlled flight into terrain as it alerts the controller when the aircraft comes in close proximity to terrain or obstacles. MSAW works with data from surveillance systems and it needs to have access to a terrain database. As the system checks the aircraft's altitude, it must get information on QNH and temperature.

The conflict is detected from the extrapolated trajectory which is based on the current track and speed in horizontal plane and on current altitude and vertical speed in vertical plane. When the vectors intersect the defined boundary (a vertical margin above the terrain), the alert is displayed.

\subsubsection{Area Proximity Warning}

Area Proximity Warning (APW) is designed to generate an alert when an unauthorised penetration of airspace volume is about to happen. The system can produce the alert when a civil aircraft is about to enter a restricted area, military aerobatic area or danger area or when an aircraft not under ATC has entered controlled airspace [8].

Similar to MSAW, the conflict is detected from the current speed and track in the horizontal plane and from the current flight level or altitude and vertical speed in vertical plane. When the horizontal and vertical boundaries of the restricted airspace volume are breached, the controller is alerted.

\section{Discussion}

The Trajectory Prediction is the core of all tools mentioned. Thanks to the system, the controllers know the accurate future position of an aircraft. The development of the prediction is the most difficult one as the aircraft movement is affected by many factors which are not easy to predict.

MTCD works with the data obtained from the trajectory prediction and calculates whether any pair of aircraft breaches the separation limits in the next 20 minutes. Although the 20 minutes horizon is required in the MTCD Specification, the research of S. Alam [9] shows it is convenient to shorten the time horizon to 15 minutes. The reason is that the number of false alerts rises very quickly between the 15 th and the 20th minute. As it is shown above, DFS already installed the new system with the 15 minutes horizon.

AMAN and DMAN are systems to optimise the landing and departing sequence of aircraft. It allows to decrease the number of planes in holding patterns, to decrease the fuel consumption by supporting the continuous descent approach, to decrease the noise pollution around airports, to decrease the number of delayed flights, to increase the runway capacity and to increase the capacity of TMA. It is recommended to implement both systems integrated so that they can together cooperate and calculate the most effective landing and departing sequence.

Safety Nets are already implemented in the air traffic control systems. It is assumed that their function will be replaced by new tools in the future. As an example, MTCD will be able to replace APW as it has the function to detect the aircraft-airspace conflicts. 


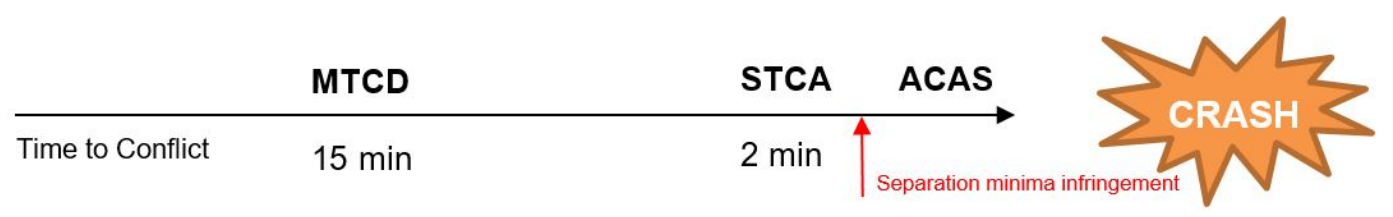

Figure 2. Order of the tools how they are activated according to the time remaining to the conflict.

\section{Conclusion}

This paper shows the major characteristics of tools, which should help the air traffic controllers to decrease their workload and to manage the current rising number of flying aircraft. It is understandable that it will take some time to develop accurate algorithms to get the best of this tools. Some of them are already implemented in the control centres which are also mentioned above. The tools are the core of the modern air traffic management. The paper shows the potential contributions of the tools and it gives recommendations for the further development.

\section{Acknowledgments}

This paper was supported by the Grant Agency of the Czech Technical University in Prague, grant No. SGS14/212/OHK2/ 3T/16

\section{References}

[1] A. Cook. European air traffic management : principles, practice and research. Routledge, London, 2007. ISBN 978-0-7546-7295-1.

[2] EUROCONTROL. Specification for MediumTerm Conflict Detection, 2010. URL http: / / www. eurocontrol.int/sites/ default/files/publication/files/ 20100715-mtcd-spec-v1.0.pdf. [Online].

[3] S. Kauppinen, C. Brain, and M. Moore. European mediumterm conflict detection field trials [ATC]. In Proceedings. The 21st Digital Avionics Systems Conference, pages 112. Institute of Electrical \& Electronics Engineers (IEEE), 2002. doi: 10.1109/dasc.2002.1067918. URL https: //doi.org/10.1109\%2Fdasc.2002.1067918.

[4] EUROCONTROL. MTCD Concept of Operation EATCHIP III Evaluation and Demonstration, 1999. URL https://www.eurocontrol.int/eec/ public/standard_page/DOC_Report_2000_ 028. html. [Online].

[5] A. Vink, S. Kauppinen, J. Beers, and K. Jong. Medium term conflict detection in EATCHIP phase III. In 16th DASC. AIAA/IEEE Digital Avionics Systems Conference. Reflections to the Future. Proceedings, pages 45-52. Institute of Electrical \& Electronics Engineers (IEEE), 1997. doi: 10.1109/dasc.1997.637322. URL https://doi . org/10.1109\%2Fdasc.1997.637322.

[6] THALES group. Maestro, 2013. URL https : / / www . thalesgroup.com/sites/default/files/ asset/document/maestro_datasheet.pdf. [Online].

[7] EUROCONTROL. Safety Nets, 2009. URL http: / / www. eurocontrol. int/safety-nets. [Online].

[8] EUROCONTROL. Guidance Material for Area Proximity Warning, 2009. URL http://www . eurocontrol. int/sites/default/files/publication/ files/20090519-apw-guid-v1.0.pdf. [Online].

[9] S. Alam, H.A. Abbass, C.J. Lokan, M. Ellejmi, and S. Kirby. Computational red teaming to investigate failure patterns in medium term conflict detection. In 8th Eurocontrol Innovation Research Workshop, Brtigny-sur-Orge, France. Eurocontrol Experimental Center. 